\title{
Effect of phason strain on the transition of an octagonal quasicrystal to a $\beta$-Mn-type structure
}

\author{
Z. H. Mai \\ Institute of Physics, Chinese Academy of Sciences, Beijing 100080, China \\ L. Xu \\ Department of Applied Physics, Beijing Polytechnic University, Beijing 100022, China
}

N. Wang and K. H. Kuo

Beijing Laboratory of Electron Microscopy, Chinese Academy of Sciences, Beijing 100080, China

Z. C. Jin and G. Cheng

Department of Applied Physics, Beijing Polytechnic University, Beijing 100022, China

(Received 20 June 1989)

\begin{abstract}
The process of transition from an octagonal quasicrystal to a crystal with $\beta$-Mn-type structure in $\mathrm{Cr}-\mathrm{Ni}$-Si alloy was investigated theoretically and experimentally. Using the linear phason strain field theory, the computer simulation of the diffraction patterns described the process of transition. The results of electron-diffraction experiments confirmed the correctness of the theoretical ones. The real-space tilings were also given. From the point of view of the geometric structural description, they also show the transition from octagonal quasiperiodicity to periodicity in a $\beta$-Mn-type structure. The transition involves the nucleation of regions of local translational periodicity in the original quasicrystal.
\end{abstract}

Since the discovery of the icosahedral quasicrystal, ${ }^{1}$ quasicrystals with tenfold and twelvefold symmetries were also reported. A new two-dimensional quasicrystal with eightfold rotational symmetry in $\mathrm{Cr}-\mathrm{Ni}-\mathrm{Si}$ and V-Ni-Si alloys has been found. ${ }^{2}$ This type of quasicrystal has also been observed in a $\mathrm{Mn}_{4} \mathrm{Si}$ alloy. ${ }^{3}$ When alloyed with a small amount of $\mathrm{Al}$, the $\mathrm{Mn}_{4}(\mathrm{Si}, \mathrm{Al})$ quasicrystal is more perfect than that of the Cr-Ni-Si alloy and it is thus possible to determine the symmetry of the octagonal phase, the noncrystallographic point group $8 / \mathrm{mmm}$, or $8 / \mathrm{m}^{4}$ High-resolution electron microscopy (HREM) observation shows that $45^{\circ}$ twins of $\beta$-Mn-type structure always coexist with the octagonal phase in the $\mathrm{Cr}-\mathrm{Ni}-\mathrm{Si}$ alloy, suggesting that these structures are closely related.

$X$-ray and electron-diffraction experiments on the icosahedral quasicrystal in Al-Mn, Al-Cu-Li, and Al-Mn-Si alloys ${ }^{5,6}$ show the distortions of symmetry in their diffraction patterns. These distortions were explained by the phason strain fields. ${ }^{7}$ Recently, it has been reported that in the $\mathrm{Al}-\mathrm{Cu}-\mathrm{Li}$ alloy the symmetry of the quasicrystal has an $m 3$ point-group symmetry, ${ }^{8}$ which deviated from the symmetry of a perfect icosahedral, and can be explained by use of a linear phason strain field. It is expected that the quasicrystal is able to be transformed into a crystal phase under an action of the phason strain field. ${ }^{9}$

Distortions of octagonal quasicrystals have also been found. Simulations by Wang and Kuo, ${ }^{10}$ using a dual transform in which the irrational number $\sqrt{2}$ was replaced with a rational approximate, produced diffraction patterns with a broken eightfold symmetry which tends to a pseudofourfold symmetry. In this paper, the transi- tion from an octagonal quasicrystal to a crystal with $\beta$-Mn-type structure is described using the linear phason strain field theory. The results of electron-diffraction experiments confirm the correctness of theoretical ones.

Based on the projection method, ${ }^{11}$ the higherdimensional space $D$ is projected into the $D \|$ subspace and its orthogonal subspace $D^{\perp}$, respectively, that is, $D=D^{\|}+D^{\perp}$. Let $R$ denote a lattice vector of a hypercubic lattice. The density is given by

$$
\rho(\mathbf{x})=\sum_{\mathbf{R}} \delta(\mathbf{x}-\mathbf{R})
$$

One can choose a window function $W(\mathbf{x})$ (Ref. 12) which cuts a slab "parallel" to the $D^{\|}$subspace in the hypercubic lattice, and place the window at a point $y=y^{\perp}(x)$. Then define a density in the $D^{\|}$subspace

$$
\begin{aligned}
\rho\left(\mathbf{x}^{\|}\right) & =\int d \mathbf{x}^{\perp} \rho(\mathbf{x}) W(\mathbf{x}-\mathbf{y}) \\
& =\sum_{\mathbf{R}} \delta\left(\mathbf{x}^{\|}-\mathbf{R}^{\|}\right) W(\mathbf{R}-\mathbf{y}), \\
W(\mathbf{x}) & = \begin{cases}1, & \mathbf{x} \in \Omega \\
0, & \text { otherwise },\end{cases}
\end{aligned}
$$

where $\Omega$ is the volume of the window. If the slab selected by the window has an "irrational" orientation, Eq. (2) specifies the density of a quasilattice.

Following the same approach used in the case of a crystal, the reciprocal space $G$ can be constructed, and projected into the two subspaces $G^{\|}$and $G^{\perp}$, namely, $G=G^{\|}+G^{\perp}$. 
The Fourier transform ${ }^{12}$ of Eq. (2) is

$$
\rho\left(\mathbf{g}^{\|}\right)=\sum_{\mathbf{G}} W^{*}\left(\mathbf{G}^{\perp}\right) \delta\left(\mathbf{g}^{\|}-\mathbf{G}^{\|}\right) \exp \left(-i 2 \pi \mathbf{G}^{\perp} \cdot \mathbf{y}^{\perp}\right) .
$$

According to the phason strain field theory, ${ }^{7}$ the density wave amplitudes are given by

$$
\rho\left(\mathbf{G}^{\|}\right)=\left|\rho\left(\mathbf{G}^{\|}\right)\right| \exp \left[i 2 \pi\left(A M \mathbf{G}^{\perp} \cdot \mathbf{R}^{\|}\right)\right],
$$

where $M$ is a second-rank tensor, and $A$ is a constant which represents the field strength of the linear phason strain. Comparing Eq. (4) with the Eq. (5), one obtains

$$
\begin{aligned}
& -\mathbf{G}^{\perp} \cdot \mathbf{y}^{\perp}=A M \mathbf{G}^{\perp} \cdot \mathbf{R}^{\perp}=\mathbf{G} \cdot A M^{-1} \mathbf{R}^{\|}, \\
& \mathbf{y}^{\perp}=-A M^{-1} \mathbf{R}^{\|} \in D^{\perp} .
\end{aligned}
$$

So Eqs. (2) and (4) can be written as

$$
\begin{aligned}
& \rho\left(\mathbf{X}^{\|}\right)=\sum_{\mathbf{R}} W\left(\mathbf{R}^{\perp}+A M^{-1} \mathbf{R}^{\|}\right) \delta\left(\mathbf{X}^{\|}-\mathbf{R}^{\|}\right), \\
& \rho\left(\mathbf{g}^{\|}\right)=\sum_{\mathbf{G}} W^{*}\left(\mathbf{G}^{\perp}\right) \delta\left(\mathbf{g}^{\|}-\left(\mathbf{G}^{\|}+A M \mathbf{G}^{\perp}\right)\right) .
\end{aligned}
$$

From the Eqs. (7) and (8), one can obtain the real-space tilings and the diffraction patterns of the quasicrystal respectively. Since both Eqs. (7) and (8) contain the term of $A M$, this means that under an action of the phason strain field, the real-space tilings and the diffraction patterns will deviate from those of a perfect quasicrystal.

In our experiments, the higher-dimensional hypercubic lattice corresponding to the octagonal quasicrystal is a four-dimensional lattice, ${ }^{10}$ the four basis vectors of $D^{\|}$are

$$
\begin{aligned}
& \mathbf{e}_{1}^{\|}=1 / \sqrt{2}(1,0,0,0), \\
& \mathbf{e}_{2}^{\|}=1 / \sqrt{2}(1 / \sqrt{2}, 1 / \sqrt{2}, 0,0), \\
& \mathbf{e}_{3}^{\|}=1 / \sqrt{2}(0,1,0,0), \\
& \mathbf{e}_{4}^{\|}=1 / \sqrt{2}(-1 / \sqrt{2}, 1 / \sqrt{2}, 0,0),
\end{aligned}
$$

and those of the $D^{\perp}$ are

$$
\begin{aligned}
& \mathbf{e}_{1}^{\perp}=1 / \sqrt{2}(0,0,1,0), \\
& \mathbf{e}_{2}^{\perp}=1 / \sqrt{2}(0,0,-1 / \sqrt{2}, 1 / \sqrt{2}), \\
& \mathbf{e}_{3}^{1}=1 / \sqrt{2}(0,0,0,-1), \\
& \mathbf{e}_{4}^{\perp}=1 / \sqrt{2}(0,0,1 / \sqrt{2}, 1 / \sqrt{2}),
\end{aligned}
$$

we have

$$
\mathbf{R}^{\|}=\sum_{i}^{4} m_{i} \mathbf{e}_{i}^{\|}, \quad \mathbf{R}^{\perp}=\sum_{i}^{4} m_{i} \mathbf{e}_{i}^{\perp},
$$

where $\left\{m_{i}\right\}$ are integers. The basis vectors of the reciprocal lattice are the same as those of the real lattice, namely,

$$
\begin{aligned}
& \mathbf{e}_{i}^{* \|}=\mathbf{e}_{i}^{\|}, \quad \mathbf{e}_{i}^{* \perp}=\mathbf{e}_{i}^{\perp}, \quad i=1, \ldots, 4 \\
& \mathbf{G}^{\|}=\sum_{i}^{4} m_{i} \mathbf{e}_{i}^{* \|}, \quad \mathbf{G}^{\perp}=\sum_{i}^{4} m_{i} \mathbf{e}_{i}^{* \perp} .
\end{aligned}
$$

Based on our experimental results, we obtained

$$
M=\left|\begin{array}{llll}
0 & 0 & 0 & 1 \\
0 & 0 & 1 & 0 \\
0 & 1 & 0 & 0 \\
1 & 0 & 0 & 0
\end{array}\right|
$$

Therefore it is possible to simulate the electrondiffraction patterns (EDP's) with different values of $A$ by computer.

Figure 1 shows the computer simulation of the diffraction patterns (ignoring the change of the diffraction intensities). One can see that due to the action of phason field, the diffraction spots calculated shift (indicated by arrows) and rotate around the ordinal eightfold axis [Fig. 1(b)]. Comparing Fig. 1(a) with 1(b), it is clear that the eightfold symmetry of the perfect octagonal quasicrystal is broken into a fourfold symmetry, but there is no translational periodicity. With the increase of the phason strain field strength, i.e., the increase of the value of the constant $A$, the diffraction spots calculated shift further, and tend to aggregate into clusters [in Figs. 1(c) and 1(d)]. When the value of $A$ reaches $\sqrt{2}-1$, the spots in each cluster are superposed upon a single point, so that the diffraction pattern has a translational periodicity [Fig.

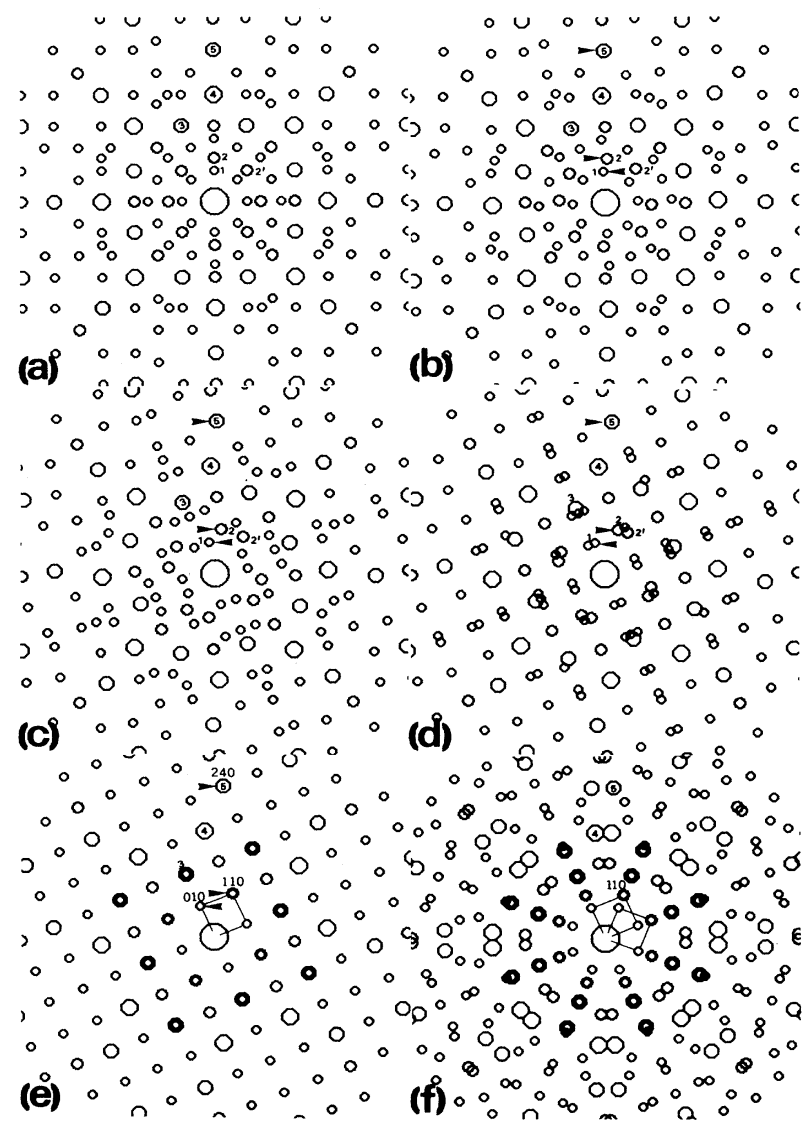

FIG. 1. Computer simulations of the electron-diffraction patterns. (a) $A=0$, (b) $A=0.05$, (c) $A=0.15$, (d) $A=0.3$, (e) $A=\sqrt{2}-1$, (f) $45^{\circ}$ overlapping pattern of (e). 
$1(\mathrm{e})]$.

It is obvious to see from Fig. 1 that under an action of phason strain field, the results of the simulated diffraction patterns show the process of transition from an octagonal quasicrystal to a crystalline structure, i.e., from quasiperiodicity with the eightfold rotation symmetry to a periodicity with fourfold rotation symmetry.

Based on the Eq. (8) the real-space tiling consisting of squares and $45^{\circ}$ rhombi can be constructed. Figure 2 shows the tilings with different values of $A$. From Figs. 2(b) and 2(c) one can see that local areas having translational periodicity (indicated by arrows) appear. More surprising is that the crystallographic structure in these areas is the same as that of the $\beta$-Mn-type structure. ${ }^{13}$ To investigate the growth of the periodic areas, a series of computer simulations has been done. As a result, with the increase of the value of constant $A$ the size of these areas grows. When the value of $A$ reaches $\sqrt{2}-1$, these periodic structures grow arbitrarily large [Fig. 2(d)]. Note that the value $A=\sqrt{2}-1$ is used in both Fig. 1(e) and Fig. 2(d). Alternately, under the action of a phason strain field with the strength $A=\sqrt{2}-1$, the octagonal quasicrystal is transformed completely into a crystal with the $\beta$-Mn-type structure. This local periodicity has been observed by means of HREM. ${ }^{13}$

To confirm the theoretical results mentioned above, the quasicrystals were examined by electron-diffraction method. The alloy films used in this investigation were prepared first by melting pure metals and silicon in an Ar arc furnace, followed by remelting and rapid solidification (about $10^{6} \mathrm{C} / \mathrm{sec}$ ) by the piston-and-anvil method. The foils suitable for transmission electron microscopy were prepared from these films by electrolytical thinning in $5 \% \mathrm{HClO}_{4}$ in ethanol at $-30^{\circ} \mathrm{C}$ followed by a brief ion thinning. They were examined in a Philips EM420 electron microscope. The size of the region from which the EDP's were taken was about $0.5 \mu \mathrm{m}$. The EDP's of quasicrystals in rapidly quenched $\mathrm{Cr}-\mathrm{Ni}$-Si alloy were taken one by one by moving the selected area aper-



FIG. 2. Computer simulations of real-space tiling. (a) $A=0$, (b) $A=0.1$, (c) $A=0.2$, (d) $A=\sqrt{2}-1$. ture or specimen. Figure 3 shows the process of the transition from the octagonal quasicrystal to the $\beta$-Mn-type structure. From Fig. 3 one can see that, because of the difference of the strength of the linear phason strain, the EDP's taken in slightly different positions are different. Comparing Fig. 1 with Fig. 3, it is obvious that the computer simulation patterns are in good agreement with the experimental ones. Certain spots move together. For example, spots 2 and $2^{\prime}$ approach each other in Figs. 3(b) and 3(c), and finally the $(001)^{*}$ diffraction pattern of $\beta$-Mn-type structure is formed [Fig. 3(d)].

Following Socolar's idea, ${ }^{14}$ the phason strain field has the same symmetry as that of the quasicrystal concerned. In the present case, we suppose that there exist two phason strain fields intersecting at $45^{\circ}$ and quenched in the octagonal phase, and that they act on the quasicrystal in equal probabilities. Therefore $45^{\circ}$ twins of $\beta$-Mn-type structure should be induced and coexist with the octagonal phase. This phenomenon has been observed experimentally by means of EDP's and HERM..$^{3,4}$ Figure 1(f) is a computer simulation superposing the patterns of Fig. $1(\mathrm{e})$ at $45^{\circ}$, it fits the experimental pattern well.

We conclude with the following remarks.

(1) Recently, we have derived theoretically that an action of phason strain drives a transition from the icosahedral quasicrystal to the cubic crystal structure. ${ }^{9}$ Now we further prove a transition from the octagonal quasicrystal to a crystal with $\beta$-Mn-type structure.

(2) The process of transition from the octagonal quasicrystal to the crystal with $\beta$-Mn-type structure in a $\mathrm{Cr}-\mathrm{Ni}-\mathrm{Si}$ alloy is explained by the linear phason field theory. The computer simulations of EDP's agree well

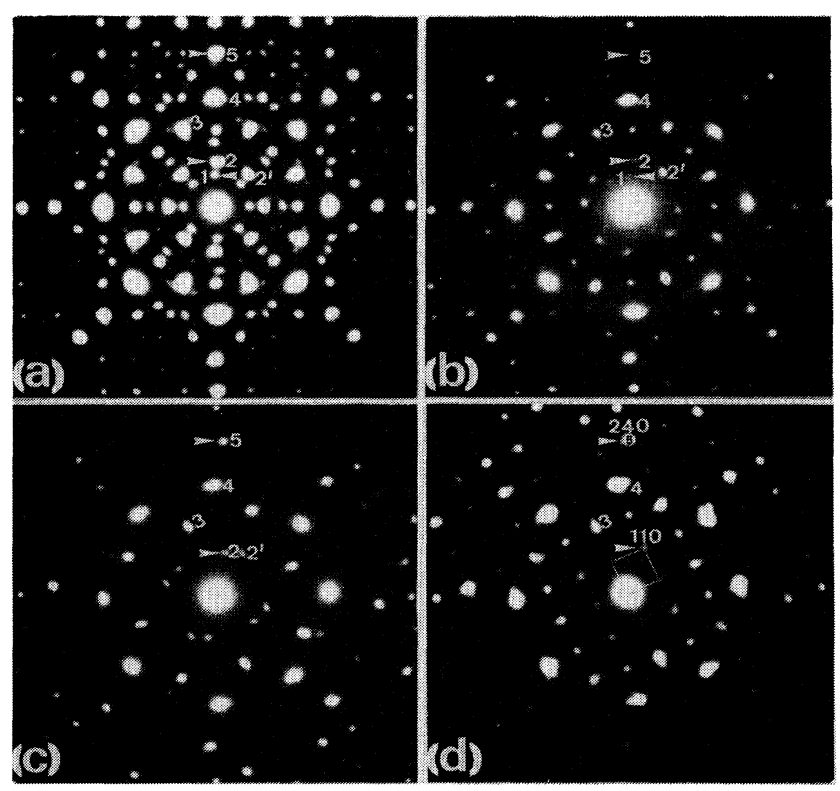

FIG. 3. The electron-diffraction patterns of Cr-Ni-Si alloy. (a), (b), and (c) the intermediate phase; (d) a $\beta$-Mn-type structure. 
with the experimental ones.

(3) The EDP's reveal that there exists an intermediate quasicrystalline structure between the perfect quasicrystal and its correlative crystal. From the point of view of the geometric structural description, the real-space tilings also show the process of transition from the octagonal quasicrystal to the crystal with $\beta$-Mn-type structure.
They reveal that the action of the phason strain field seems to be that the $\beta$-Mn-type structure nucleates in the octagonal quasicrystal with relative orientation.

The authors would like to thank professor K. K. Fung, Dr. B. S. Zhang, and Mr. Z. R. Huang for helpful discussions.
${ }^{1}$ D. Shechtman, I. Blech, D. Gratias, and J. W. Cahn, Phys. Rev. Lett. 53, 1951 (1984).

${ }^{2}$ N. Wang, and K. H. Kuo, Phys. Rev. Lett. 59, 1010 (1987).

${ }^{3}$ W. Cao, H. Q. Ye, and K. H. Kuo, Phys. Status Solidi 107, 511 (1988).

${ }^{4}$ N. Wang, K. K. Fung, and K. H. Kuo, Appl. Phys. Lett. 52, 2120 (1988).

${ }^{5}$ P. A. Bancel, P. A. Heiney, P. W. Stephens, I. Goldman, and P. M. Horn, Phys. Rev. Lett. 54, 2422 (1985).

${ }^{6}$ Z. H. Mai, B. S. Zhang, M. J. Hui, Z. H. Huang, and X. S. Chen, Mater. Sci. Forum 22-24, 591 (1987).

${ }^{7}$ T. C. Lubensky, J. E. S. Socolar, P. J. Steinhardt, P. A. Bancel, and P. A. Heiney, Phys. Rev. Lett. 57, 1440 (1986).

${ }^{8}$ Z. H. Mai, S. Z. Tao, L. Z. Zeng, and B. S. Zhang, Phys. Rev. B 38, 12913 (1988).

${ }^{9}$ Z. H. Mai, S. Z. Tao, B. S. Zhang, and L. Z. Zeng, J. Phys. C 1, 2465 (1989).

${ }^{10}$ Z. M. Wang and K. H. Kuo, Acta Cryst. A 44, 857 (1988).

${ }^{11}$ V. Elser, Phys. Rev. B 32, 4892 (1985); Acta Cryst. A 42, 36 (1986).

${ }^{12}$ M. V. Jarić, Phys. Rev. B 34, 4685 (1986).

${ }^{13}$ N. Wang and K. H. Kuo, Philos. Mag. (to be published).

${ }^{14}$ J. E. S. Socolar and D. C. Wright, Phys. Rev. Lett. 59, 221 (1987). 


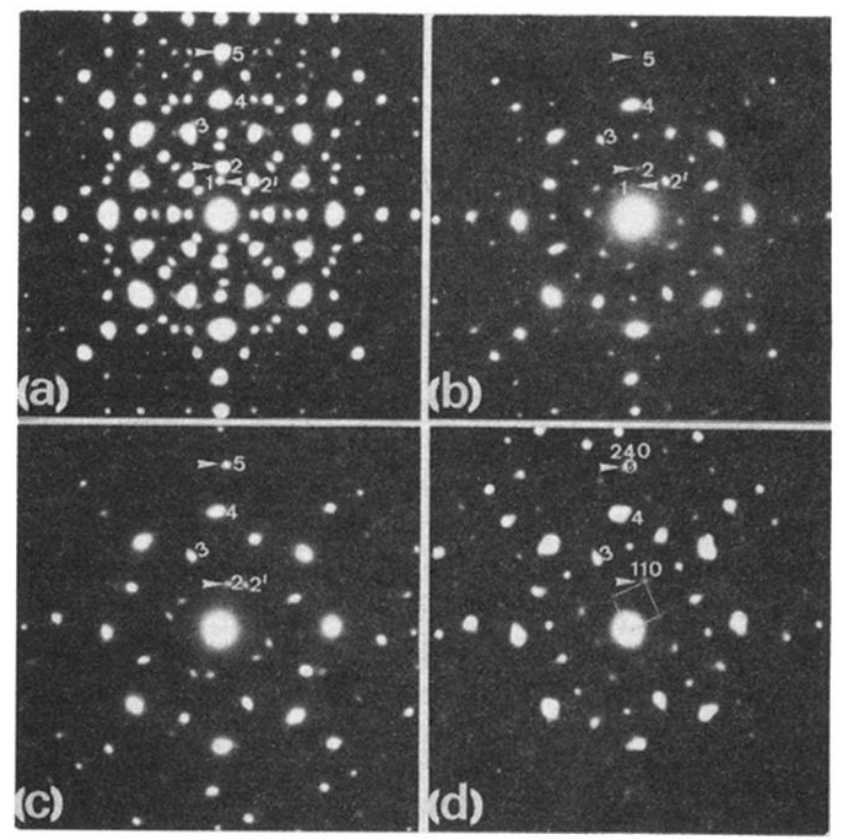

FIG. 3. The electron-diffraction patterns of $\mathrm{Cr}-\mathrm{Ni}-\mathrm{Si}$ alloy. (a), (b), and (c) the intermediate phase; (d) a $\beta$-Mn-type structure. 